FORMATLON Formation emploi

Revue française de sciences sociales

131 | Juillet-Septembre 2015

Le Bac Pro a 30 ans

\title{
Le poids des relations familiales dans la mobilité géographique des jeunes inscrits en baccalauréat professionnel
}

The weight of family relathionships in the geographical mobility of young people registered for vocational baccalaureate.

Das Gewicht familiärer Beziehungen in der geografischen Mobilität von

Berufsabitur-Kandidaten

El peso de las relaciones familiares en la movilidad geográfica de los jóvenes inscriptos en el bachillerato técnico

\section{Alain Thalineau et Florian Hot}

\section{(2) OpenEdition}

\section{Journals}

Édition électronique

URL : http://journals.openedition.org/formationemploi/4495

DOI : 10.4000/formationemploi.4495

ISSN : 2107-0946

Éditeur

La Documentation française

Édition imprimée

Date de publication : 15 octobre 2015

Pagination : 101-120

ISSN : 0759-6340

Référence électronique

Alain Thalineau et Florian Hot, «Le poids des relations familiales dans la mobilité géographique des jeunes inscrits en baccalauréat professionnel », Formation emploi [En ligne], 131 | Juillet-Septembre 2015, mis en ligne le 15 octobre 2017, consulté le 30 octobre 2020. URL : http:// journals.openedition.org/formationemploi/4495 ; DOI : https://doi.org/10.4000/formationemploi.4495 


\title{
Le poids des relations familiales dans la mobilité géographique des jeunes inscrits en baccalauréat professionnel
}

\author{
Alain Thalineau \\ Sociologue, professeur des universités, UMR CITERES (Cités, Territoires, Environnement et \\ Sociétés) 7324, université de Tours, CNRS \\ FLORIAN HOT \\ Sociologue, ingénieur d'études, UMR CITERES (Cités, Territoires, Environnement \\ et Sociétés) 7324, université de Tours, CNRS
}

Résumé

Le poids des relations familiales dans la mobilité géographique des jeunes inscrits en baccalauréat professionnel

A l'heure où la mobilité (résidentielle et quotidienne) s'impose comme une qualité indispensable à tout individu visant son insertion sur le marché de l'emploi, comment les lycéens inscrits en baccalauréat professionnel en région Centre envisagent-ils l'éventualité d'un départ et d'un éloignement du groupe familial pour obtenir un premier emploi ? L'enquête quantitative menée auprès de 536 lycéens et l'analyse de 55 entretiens biographiques mettent en évidence le lien entre une aspiration à la mobilité professionnelle, l'intensité des relations au groupe familial, et la posture des parents vis-à-vis de la norme scolaire.

Mots clés : jeunes, mobilité géographique, insertion professionnelle, famille, baccalauréat professionnel.

Abstract

The weight of family relathionships in the geographical mobility of young people registered for vocational baccalaureate.

Nowadays, mobility (both daily and residential) prevails as a must-have asset for any individual aiming for their occupational integration. But how do the students taking vocational baccalaureate in the Centre region consider the possibility of a departure or an estrangement from the family group to get a first job? A quantitative survey has been carried out on 536 students and an analysis of 55 biographical interviews has been achieved. They both give prominence to a connection between an aspiration for 
professional mobility, the strength of relationships towards the family group and the position of parents regarding academic standards.

Keywords: young person, geographic mobility, transition from school to work, family, vocational baccalaureate.

Journal of Economic Literature: $\mathbf{J} \mathbf{6 1}$

Traduction : Auteurs

La mobilité des individus s'est imposée comme une valeur centrale de notre société. Associée à l'aventure, la liberté, l'épanouissement personnel... Elle est également, et non sans lien, un des traits caractéristiques du développement capitaliste. En matière d'emploi, la mobilité est souvent présentée comme une nécessité lors de l'insertion professionnelle (Freyssinet, 2004), mais également pour rester dans l'emploi.

La mobilité des travailleurs, le plus souvent subie depuis les débuts du capitalisme (Geremek, 1987 ; Castel, 1989, 1995), suit les mouvements du marché économique et constitue un enjeu majeur des politiques de gestion de la main-d'œuvre.

Elle s'accompagne d'une mobilité résidentielle qui devient, dans le discours dominant, une condition nécessaire pour être en mesure de se maintenir dans l'emploi.

Les jeunes, en tant que public cible des politiques de lutte contre le chômage depuis le début des années 80 , font particulièrement l'objet de ces injonctions. Ils multiplient les expériences de mobilité géographique qui devient une norme sociale.

Si le phénomène n'est pas nouveau, il s'est développé chez les jeunes adultes au cours des dernières décennies (Baron M., Perret C., 2006). La mobilité est considérée comme positive et créatrice d'expériences valorisantes et valorisables dans des logiques d'insertion sociale et professionnelle. Dans les faits, elle favorise "l'insertion professionnelle " (Perret, 2007).

L'école, désormais chargée de favoriser "linsertion professionnelle " (Geay, 2003), se doit d'être au premier rang pour socialiser les jeunes afin qu'ils soient les mieux " adaptés " aux exigences du marché de l'emploi. Les lycéens suivant des formations professionnalisantes courtes, de type baccalauréat professionnel, tendent à s'orienter vers l'emploi au terme de leur cursus. La tendance est à l'allongement de la durée des études, depuis la seconde explosion scolaire déclenchée en 1985 par le projet de mener $80 \%$ d'une classe d'âge au niveau du baccalauréat, ce qui coïncide avec la création du baccalauréat professionnel ; pour autant, actuellement, près de $75 \%$ des jeunes lycéens en formations professionnelles ne poursuivent pas d'études dans l'enseignement supérieur après le baccalauréat ${ }^{1}$ (Péan, 2012). La question de la mobilité résidentielle pour l'emploi leur est donc posée de manière

1. Résultats relatifs à la France métropolitaine ainsi que ses Départements d'Outre Mer. 
précoce. Comment, en amont de leur entrée prochaine sur le marché de l'emploi, envisagent-ils l'éventualité d'un changement de résidence et d'un éloignement du groupe familial pour obtenir un premier emploi ? Cela interroge la construction sociale de la mobilité professionnelle chez des jeunes qui, d'une part, en raison de leur âge, ont peu d'expériences d'éloignement du « nid " familial et, d'autre part, sont tendanciellement de milieux populaires, c'est-à-dire de milieux d'ouvriers et d'employés, en position dominée tant sur le plan économique que culturel, confrontés de façon accrue à l'insécurité économique².

Pour saisir le rôle que joue le groupe familial dans la construction de l'aspiration à la mobilité pour l'emploi des jeunes scolarisés en baccalauréat professionnel, les données ont été recueillies auprès de jeunes en formation dans des zones urbaines et rurales de la région Centre ${ }^{3}$, et dans des établissements scolaires proposant des formations diversifiées.

Des informations recueillies par questionnaires auto-administrés ( $c f$. encadré 1), il ressort que les lycéens scolarisés en baccalauréat professionnel ne sont pas nécessairement favorables à l'éventualité d'une mobilité pour trouver un emploi, et ce, quel que soit le lieu où ils sont en formation.

L’enquête complémentaire par entretien révèle que les jeunes ayant arrêté leur formation avant le baccalauréat sont, par ailleurs, plus rétifs à la mobilité que les jeunes scolarisés, quel que soit le lieu de leur formation. Les variations sont liées, d'une part, à la façon dont le jeune et ses parents se situent vis-à-vis des attentes de l'école et du monde du travail et, d'autre part, à la cohésion du groupe familial.

Ainsi, deux pôles s'opposent de façon dominante : celui du groupe familial ayant une forte cohésion et où les parents ont un emploi stable et sont porteurs de la norme scolaire ; celui du groupe familial en situation de vulnérabilité sociale (Castel, 1991), dont les parents sont éloignés de la norme scolaire et de la norme emploi ; l'ensemble des situations rencontrées se situant plus ou moins vers l'un ou l'autre de ces pôles en fonction des positions sociales occupées par les parents et de la cohésion du groupe familial. Après une présentation des caractéristiques sociales des différentes projections de mobilité professionnelle au sein de la population interrogée, le propos consistera à caractériser ces deux pôles.

2. Il ne s'agit pas ici de nier la diversité des positions au sein même des milieux populaires, ni le fait que tous les jeunes en lycée professionnel ne sont pas de milieux populaires, mais, bien au contraire, de prendre en compte la façon dont les jeunes envisagent objectivement et subjectivement la mobilité professionnelle en tenant compte des ressources économiques et culturelles du groupe familial (Mauger, 2013). On peut également avoir à l'esprit la tendance à l'obsolescence du capital d'autochtonie au sein des milieux ouvriers : "fragile, vulnérable, ce capital, qui pourrait être qualifié de capital du petit peuple intégré, subit de plein fouet une obsolescence qui n'est pas sans relation avec les processus de désaffliation sociale qui atteignent en premier chef les classes populaires et leurs espaces de résidence " (Retière, 2003, pp. 139-140).

3. Les données ont été recueillies dans le cadre du programme de recherche régional Formation et insertion professionnelle : «l'impact des ressources familiales et territoriales”, région Centre, 2011-2014. 


\section{Encadré 1 : L'enquête}

Le questionnaire produit par l'équipe de recherche avait pour objectif de fournir des informations au sujet des parcours de formation, des sociabilités, des relations à la famille, des rapports aux territoires et des projections dans l'emploi, de lycéens scolarisés en première et terminale en 2012-2013 dans 8 établissements de la Région Centre, situés sur trois zones d'emploi distinctes. II prenait la forme d'un livret comportant 76 questions.

La méthode de passation du questionnaire, est à mi-chemin entre l'administration directe et l'auto-administration. En passant par l'intermédiaire des responsables des lycées, il a été possible de mettre en place un protocole de distribution des questionnaires directement dans les classes. Cette coopération permettait de solliciter facilement un effectif important de jeunes et de limiter les non-réponses en raison de la présence d'un enseignant lors de la passation.

Sur les deux villes de Tours et Châteauroux, les lycées professionnels accueillant le plus grand nombre d'élèves ainsi que des établissements offrant des formations spécifiques ont été retenus. La commune rurale de Beaune-la-Rollande, près de Montargis, est venue s'y ajouter, car l'établissement secondaire qui s'y trouve est un lycée professionnel agricole. Ces choix ont été effectués dans le but d'étudier, au-delà de l'influence des cursus de formation, l'existence ou non de relations entre l'aspiration à la mobilité pour rechercher un emploi, les liens que le jeune entretient avec ses proches, et le rapport du groupe familial à la norme scolaire et la norme emploi, sachant par ailleurs que les types d'orientations professionnelles envisageables par les jeunes en formation dépendent non seulement du rendement des formations sur le marché de l'emploi local, mais également de l'influence des proches dans les projets à venir (Caro, 2006). Au final, 536 questionnaires ont alors été recueillis, servant de matériau à l'analyse statistique.

\section{Répartition des répondants par ville et par lycée}

\begin{tabular}{|c|c|c|c|c|}
\hline Ville & Lycée & Répondants & \% & \% / ville \\
\hline Beaune-la-Rolande (45) & Beaune-la-Rolande & 55 & $10 \%$ & \multirow{2}{*}{$10 \%$} \\
\hline \multirow{3}{*}{ Châteauroux (36) } & Blaise Pascal & 23 & $4 \%$ & \multirow{2}{*}{$29 \%$} \\
\cline { 2 - 4 } & Les Charmilles & 134 & $25 \%$ & \\
\hline \multirow{3}{*}{ Tours Agglomération (37) } & Albert Bayet & 117 & $22 \%$ & \multirow{2}{*}{$61 \%$} \\
\cline { 2 - 4 } & François Clouet & 66 & $12 \%$ & \\
\cline { 2 - 4 } & Gustave Eiffel & 49 & $9 \%$ & \\
\cline { 2 - 4 } & Martin Nadaud & 35 & $7 \%$ & \multirow{2}{*}{$100 \%$} \\
\hline & Saint-Vincent-de-Paul & 57 & $11 \%$ & \\
\hline
\end{tabular}




\section{Encadré 2 : La région Centre}

La région Centre est un territoire où les jeunes de 18 à 24 ans partent légèrement plus qu'ils ne viennent s'installer (Durville J-M et Connin P., 2009). De 2001 à 2006, le solde migratoire des 18-24 ans avait un taux négatif de $-11 \%$. Cela s'explique notamment par la proximité avec la région lle-de-France qui constitue un bassin attractif tant en matière d'offre de formation que d'insertion professionnelle. Sur le territoire régional lui-même, certaines agglomérations urbaines sont également plus attractives que d'autres.

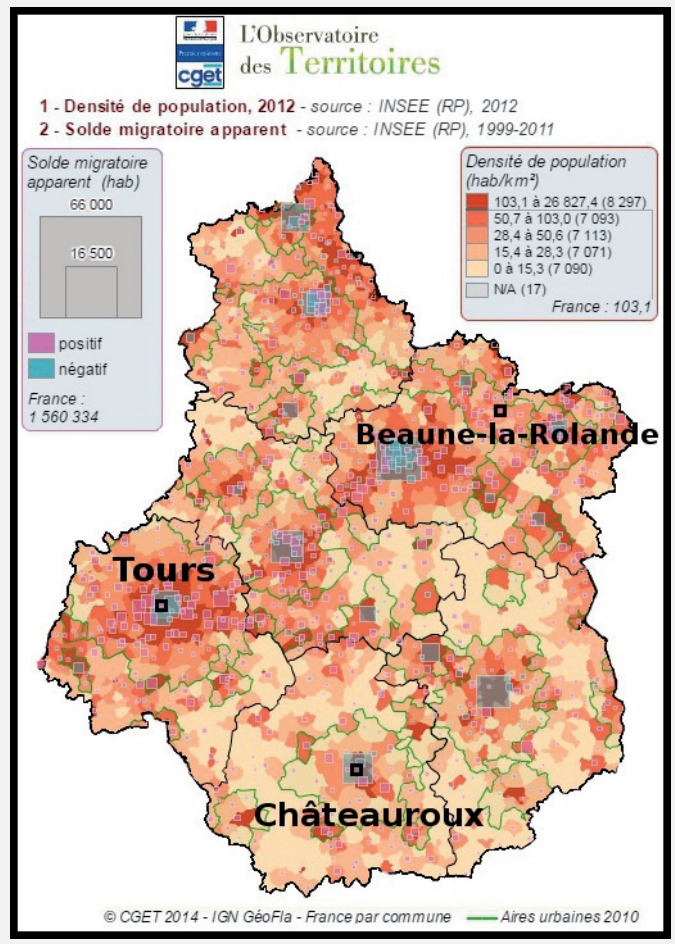

Un indicateur de ces différences est la part de jeunes en formation par rapport à l'ensemble de la population. La plus ou moins grande densité de « jeunes » sur l'espace géographique étudié peut influer sur l'attachement au territoire habité. Pour des jeunes provenant du milieu rural, scolarisés en internat dans une "ville de jeunes », les caractéristiques de la ville habitée pendant la formation peuvent avoir éventuellement un effet sur l'investissement dans la formation et dans le projet professionnel. Pour contrôler cette variable, nous avons choisi de réaliser nos investigations sur trois sites : deux fortement urbains, mais différents quant à la part des jeunes en formation dans la population, et un site en milieu rural.

La zone d'emploi de Tours se caractérise par une population en formation âgée de moins de 25 ans de l'ordre de 37,29 \% par rapport à l'ensemble de la population sur ce territoire (INSEE, données locales, 2009). Elle représente $17,5 \%$ de la population présente sur le bassin d'emploi de la ville de Châteauroux. 


\section{Les projections de mobilité résidentielle pour l'emploi diffèrent selon les liens familiaux}

Répartis dans plus de 80 spécialités, les jeunes en lycée professionnel ne bénéficient pas de conditions de formation similaires. Certaines filières sont plus demandées que d'autres ; les stages ne se réalisent pas dans le même type d'entreprise, des formations présentent une dominante masculine, d'autres féminine. Par ailleurs, les différents travaux de recherche portant sur les comportements de mobilité résidentielle des jeunes au terme de leur cursus scolaire ont souligné les différences de pratiques selon le genre, l'origine sociale et le type de formation suivi (Couet, 2006 ; Didier-Fèvre, 2014). L'échantillon de l'enquête par questionnaire a été constitué afin d'appréhender une diversité de profils de jeunes inscrits en bac professionnel sur des territoires différents et dans des filières fortement hétérogènes, tout en s'approchant des données structurelles nationales de la population. Il s'agissait de saisir, au-delà des différences de cursus de formation, l'existence ou non d'une relation entre l'aspiration à la mobilité pour rechercher un emploi, les relations que le jeune entretient avec ses proches, et les prises de position du jeune et des parents vis-à-vis de la norme scolaire et de la norme emploi.

\subsection{Des similitudes selon le genre, l'origine sociale et le domaine de formation}

En matière de répartition par sexe et milieu d'origine, l'effectif conservé présente des caractéristiques similaires à la population des inscrits au baccalauréat professionnel au niveau régional ${ }^{4}$ et national ${ }^{5}$. Quant au domaine de formation (services ou production), l'objectif étant de diversifier les filières de formation, la représentativité n'a pas été recherchée.

En 2012, sur l'ensemble des élèves préparant le baccalauréat professionnel ou un brevet des métiers d'art ${ }^{6}$ (BMA) sur le territoire national (départements d'outre-mer compris), tous niveaux confondus (seconde, première et terminale), la part des filles s'élevait à 43,6\%. Dans l'enquête par questionnaire, la répartition par genre est très proche, puisque $45 \%$ des questionnaires analysés ont été renseignés par des filles.

S'agissant de l'origine sociale des lycéens, l'échantillon est proche des grandes tendances observées aux niveaux régional et national, notamment concernant la surrepré-

4. Selon les données de l'ORFE (Observatoire Régional de la Formation et de l'Emploi)

5. Selon les données des ministères de l'Education nationale et de l'Enseignement supérieur et de la Recherche publiées dans « Repères et références statistiques 2013 ». Résultats relatifs à la France métropolitaine et aux départements d'outre-mer.

6. Les données proposées par l'éducation nationale dans "Repères et références statistiques 2013" regroupent les filières préparant au diplôme du baccalauréat professionnel et celles conduisant au Brevet des métiers d'art (BMA), diplôme rare également de niveau IV. 
sentation des fils et filles d'ouvriers. Alors que sur l'ensemble de la population active âgée de 15 à 64 ans en région Centre, on compte 27,2 \% d'ouvriers (Sources : Insee, RP2009 exploitations complémentaires), la catégorie "enfants d'ouvriers " représente $38,28 \%$ du total des inscrits en baccalauréat professionnel dans la région et $42 \%$ de notre échantillon.

Quant aux domaines de formation, l'objectif étant la diversification des cursus, l'effectif traité se répartit dans 17 filières de formation. Au niveau national, les lycéens préparant un baccalauréat professionnel (bac pro) ou un brevet des métiers d'art (BMA), sont légèrement plus nombreux dans le domaine des services $(57,9 \%)$ que dans celui de la production $(42,1 \%)^{7}$. Cette tendance est plus nette dans notre effectif, puisque $67,2 \%$ des enquêtés sont inscrits dans des formations relevant du secteur tertiaire.

\subsection{Des mobilités liées au positionnement familial vis-à-vis de la norme scolaire}

Les jeunes en lycée professionnel sont plutôt enclins à s'attacher aux lieux qui constituent leur territoire de vie au quotidien (Jellab, 2008). Pour autant, ils n'échappent pas au discours dominant sur le " devoir " d'être mobile pour trouver un emploi.

L'enquête quantitative révèle la variation des points de vue des jeunes de la région Centre quant à leur aspiration ou non à quitter le domicile parental ${ }^{8}$ pour occuper un premier emploi éloigné de ce domicile. Se disant à la fois "prêts pour partir " et " inquiets", les jeunes en lycée professionnel expriment ici un trait caractéristique de l'aspiration à l'émancipation des adolescents ( $c f$. graphiques).

L'aspiration ou non à la mobilité pour l'emploi ne dépend pas en soi des PCS et des diplômes des parents ${ }^{9}$. De même, le secteur de formation (service ou production) ne semble pas explicatif de ces résultats, puisque $58 \%$ des jeunes relevant du secteur des services se déclarent prêts au départ, contre $57 \%$ des jeunes du secteur de la production. Enfin, le genre et l'âge n'influencent pas davantage cette tendance. Confrontée à ces deux variables, la part des jeunes déclarant être prêts au départ reste relativement stable.

\footnotetext{
7. Données issues de "Repères et références statistiques 2013 ".

8. Par conséquent, les jeunes concernés par l'analyse présentée dans cet article sont ceux qui vivent au domicile parental (tout en pouvant être internes au lycée), soit un effectif de 485 jeunes.

9. Cette absence d'influence des PCS et des niveaux d'études est à relativiser en raison de la proportion des non-réponses. Concernant la PCS du père et de la mère, le taux de non-réponses est respectivement de $23 \%$ et de $20 \%$; pour le niveau d'études, il est respectivement de $30 \%$ et $27 \%$.
} 


\section{Graph 1 - Disposition à quitter leur famille}
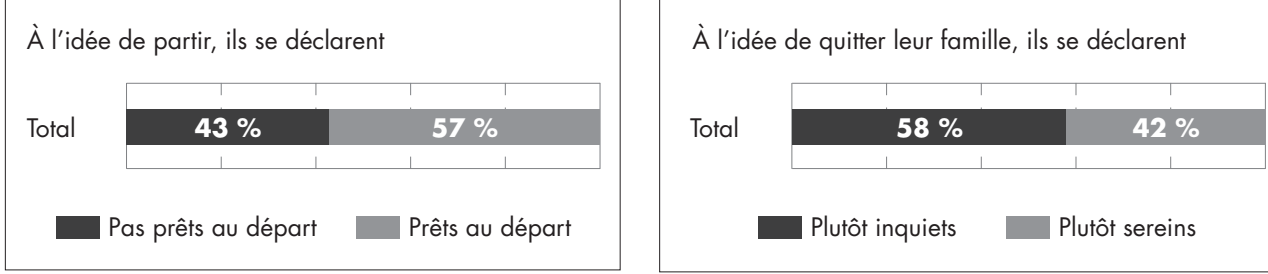

Lecture : $58 \%$ (460) des jeunes ayant répondu à la question, soit 267 individus, se disent « plutôt inquiets » de quitter le domicile familial afin d'obtenir leur premier emploi.

Source : Données recueillies dans le cadre du programme de recherche régional Formation et insertion professionnelle : « l'impact des ressources familiales et territoriales", région Centre, 2011-2014.

\section{Graph 2 - Disposition à quitter leur département}

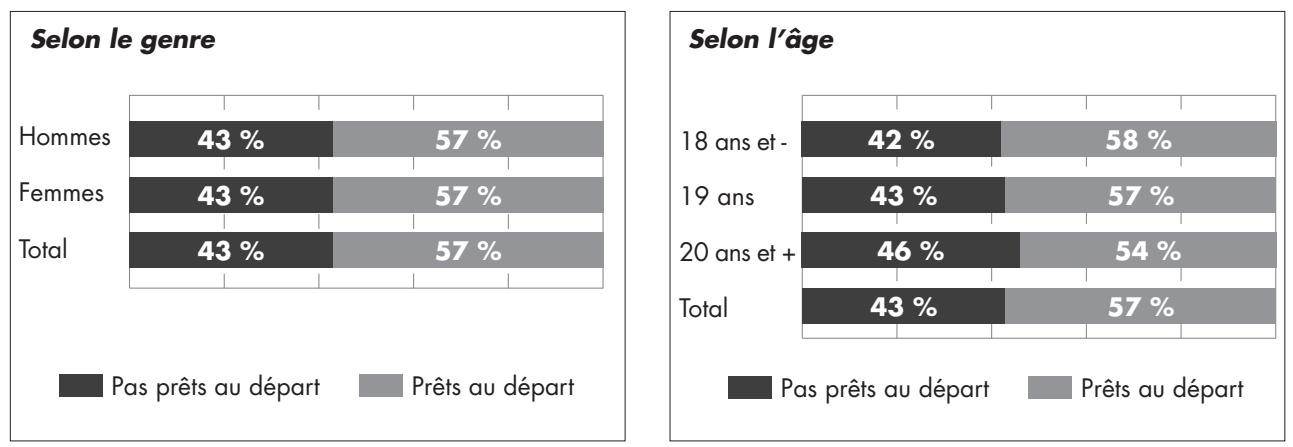

Lecture : 57 \% (463) des jeunes ayant répondu à la question ont déclaré se sentir « prêts » à quitter le département afin d'obtenir un emploi. Source : Données recueillies dans le cadre du programme de recherche régional Formation et insertion professionnelle : «L'impact des ressources familiales et territoriales", région Centre, 2011-2014.

Pour autant, cette dualité de la projection de la mobilité pour l'emploi ("inquiets ", mais "désireux de partir " pour accéder à l'emploi) prend un sens particulier chez des jeunes en lycée professionnel. Les trois quarts d'entre eux chercheront, dans les deux années, à obtenir un emploi dans un contexte économique peu favorable à l'embauche. Face à cette perspective, quels sont les ressorts sociaux de leur positionnement vis-à-vis de la mobilité résidentielle pour trouver un emploi ? L'analyse conjointe des matériaux quantitatif et qualitatif révèle que leur positionnement se situe entre deux pôles caractérisés par le type de relations qu'ils entretiennent avec leurs proches, et par le degré d'investissement du groupe familial vis-à-vis de la norme scolaire. 


\section{Rester ou partir, un enjeu pour être intégré dans le monde du travail}

Investir dans l'école "pour s'en sortir " est un enjeu majeur, même si, au sein des milieux populaires, cet investissement a un rendement limité. Lorsque le groupe familial est fortement porteur des normes et valeurs dominantes de la réussite scolaire et de la norme emploi, et lorsque les relations au sein de la famille sont paisibles, alors l'enfant est plus facilement en conformité avec le "désir-maître " des institutions (Lordon, 2010). Mais cela s'accomplit dans un tiraillement. Partir, c'est aussi quitter les proches avec qui on se sent protégé, tout particulièrement au sein des milieux sociaux où le monde extérieur apparaît désormais fragmenté, dénué de solidarité collective, et hostile (Schwartz, 1990 ; Beaud, Pialoux, 1999). Cette ambivalence apparaît dans l'analyse croisée des données quantitatives et qualitatives.

\subsection{La famille, un espace affectif rassurant}

Les types de relation que les jeunes entretiennent avec leurs parents et leur aspiration à l'émancipation on été repérés en croisant une question du questionnaire, abordant l'aspiration ou non à quitter le « nid » familial, et une autre traitant de la façon d'appréhender les relations au sein du foyer. D'où quatre types de positionnement subjectif vis-à-vis de la famille et du monde extérieur. Afin de les qualifier en tenant compte de ce rapport subjectif à la sphère familiale et au monde extérieur, ils ont été définis ainsi dans le tableau 1 :

Tableau 1 : Quatre types de positionnement subjectif vis-à-vis de la famille et du monde extérieur

\footnotetext{
Les « casaniers paisibles » $\left(30,8 \%{ }^{\circ}\right)$ déclarant bénéficier d'une « ambiance paisible » dans le foyer familial et désirant rester au domicile.

Les « aventuriers paisibles » $\left(38 \%{ }^{\circ}\right)$ déclarant bénéficier d'une « ambiance paisible » dans le foyer familial, mais désirant quitter rapidement le domicile $(*)$.

Les « casaniers en tension » $\left(6,6 \%^{\circ}\right)$ déclarant évoluer dans un climat de tensions, voire conflictuel dans le foyer familial, mais désirant rester au domicile $(* *)^{2}$.

Les « aventuriers en tension » (24,6\%) déclarant évoluer dans un climat de tensions, voire conflictuel, dans le foyer familial, et désirant quitter le domicile.

$\left.{ }^{*}\right)$ : Il est à noter ici que la modalité exprimant le souhait de partir insiste sur l'intensité de cette aspiration au départ : «j'attends avec impatience d'avoir un logement personnel «. Elle n'est pas significativement dépendante de l'âge et du sexe.

$\left({ }^{* *}\right)$ : Cette sous-population est marquée par une surréprésentation des femmes (61 \% contre $43 \%$ de l'effectif global).
} 
Concernant l'aspiration à la mobilité professionnelle, les jeunes relevant du type " casaniers paisibles » se déclarent peu désireux de s'éloigner rapidement du groupe familial pour trouver un emploi :

\section{Graph 3 - Sentiment lié à la mobilité résidentielle présumée par l'occupation du premier emploi selon la forme de la relation à la famille}

\begin{tabular}{|c|c|c|}
\hline Casaniers paisibles & $67 \%$ & $33 \%$ \\
\hline Aventuriers paisibles & $53 \%$ & $47 \%$ \\
\hline Casaniers en tension & $66 \%$ & $34 \%$ \\
\hline Aventuriers en tension & $53 \%$ & $47 \%$ \\
\hline \multirow[t]{2}{*}{ Total } & $58 \%$ & $42 \%$ \\
\hline & $t$ inquiets & Plutôt sereins \\
\hline
\end{tabular}

$\mathrm{p}=4 \% ;$ chi2 $=8,3 ; \mathrm{ddl}=3$

Lecture : Le test de chi2 réalisé avec le logiciel Sphinx montre l'existence d'une relation significative entre les deux variables ( $96 \%$ de chances de rejeter H0). 33 \% du groupe des " casaniers paisibles », contre $47 \%$ des « aventuriers paisibles » et des " aventuriers en tension », appréhendent plutôt sereinement la nécessité de quitter le domicile familial afin d'obtenir leur premier emploi.

Source : Données recueillies dans le cadre du programme de recherche régional Formation et insertion professionnelle : « l'impact des ressources familiales et territoriales", région Centre, 2011-2014.

\section{Graph 4 - Disposition à quitter le département pour trouver un emploi selon la forme} de la relation à la famille

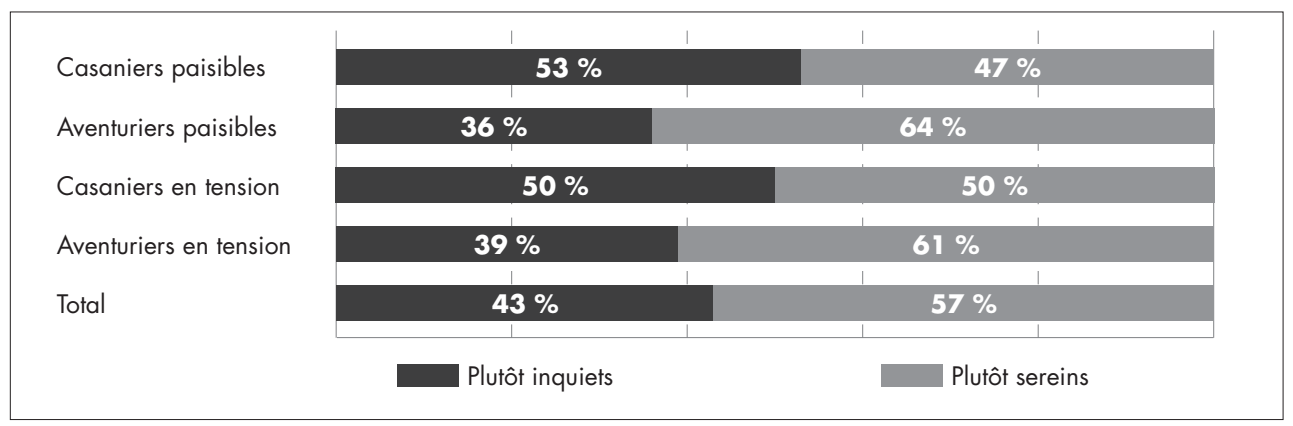

$\mathrm{p}=1 \% ;$ chi2 $=10,5 ; \mathrm{ddl}=3$

Lecture : Le test de chi2 réalisé avec le logiciel Sphinx montre l'existence d'une relation significative entre les deux variables ( $99 \%$ de chance sde rejeter H0). $53 \%$ du groupe des « casaniers paisibles » contre $36 \%$ des « aventuriers paisibles » ne se sentent pas prêts à quitter le département afin d'obtenir un emploi.

Source : Données recueillies dans le cadre du programme de recherche régional Formation et insertion professionnelle : «l'impact des ressources familiales et territoriales", région Centre, 2011-2014. 


\section{Encadré 3 : 55 récits biographiques de jeunes lycéens ou ex-lycéens}

L'enquête a permis de recueillir des récits biographiques fournissant des informations sur les parcours familiaux, scolaires, résidentiels, sur les sociabilités et les espaces fréquentés, sur le rapport au travail et à l'emploi.

Une partie des jeunes rencontrés dans le cadre de l'enquête qualitative était scolarisée en première ou terminale bac pro lors de l'entrevue. L'autre partie avait interrompu la formation en bac pro et se trouvait, lors de l'entretien, soit dans une formation en apprentissage, soit dans une formation de remise à niveau proposée par la mission locale, dans une formation à l'Ecole de la deuxième chance ou était sans formation et sans emploi $\left({ }^{*}\right)$. En choisissant ces deux populations distinctes, l'objectif était de saisir l'intrication des différents faits relevant de la sphère familiale, des relations aux groupes de pairs, des relations aux institutions scolaires, contribuant à des arrêts de formation ou au maintien dans la formation (Esterle-Hedibel, 2006). La comparaison a constitué un moyen pour repérer les forces des différentes sphères d'intégration sociale sur la construction sociale des aspirations à la mobilité professionnelle.

Les entretiens des jeunes scolarisés se sont déroulés au sein de l'institution scolaire, dans un espace neutre. Pour les jeunes qui n'étaient plus en bac pro, les entretiens ont eu lieu principalement à domicile. L'ensemble des entretiens a été analysé selon la méthode biographique (Demazière, Dubar, 1997) : les entretiens ont été retranscrits et thématisés avec le logiciel Sonal afin de permettre l'analyse structurale des récits, qui a été réalisée par l'établissement des relations d'association et d'opposition entre les différents sèmes constitutifs des énoncés thématisés (Blanchet, Gotman, 1992)

Autant d'hommes que de femmes ont été rencontrés, mais les hommes étaient plus fréquemment en situation de formation bac pro.

\begin{tabular}{|c|c|c|c|}
\hline $\begin{array}{c}\text { Scolarité en } \\
\text { BAC-PRO }\end{array}$ & Femme & Homme & Total \\
\hline Réorientation & 15 & 9 & 24 \\
\hline Scolarisé & 14 & 17 & 31 \\
\hline Total & 29 & 26 & 55 \\
\hline
\end{tabular}

$\left(^{*}\right)$ : Cette population, de part sa volatilité une fois sortie du lycée professionnel, n'a pas pu être mobilisée pour l'enquête quantitative.

Le groupe des " casaniers paisibles ", apparemment peu désireux de s'éloigner du groupe familial pour trouver un emploi, est aussi celui ayant une situation familiale stable. $73 \%$ des jeunes de ce groupe vivent au sein de familles constituées des parents biologiques, contre $56 \%$ des jeunes du groupe des " aventuriers en tension ». Par ailleurs, dans ce groupe, les parents sont les moins pourvus en capital scolaire. Cela est particulièrement significatif s'agissant du niveau de diplôme de leur mère : $63 \%$ des jeunes des groupes " casaniers " déclarent que leur mère détient un BEP (brevet d'études professionnelles), un CAP (certificat d'aptitude professionnelle), le Brevet des collèges, ou ne détient aucun diplôme. À l'inverse, pour les groupes des " aventuriers ", la majorité des jeunes (" aven- 
turiers paisibles " : $52 \%$; «aventuriers en tension " : $63 \%$ ) déclarent que leur mère possède le baccalauréat ou un diplôme d'études supérieures. Au sein d'une famille faiblement dotée pour prendre place au sein du monde extérieur (l'école, l'entreprise, l'espace du politique), le groupe familial constitue, pour ces jeunes, l'univers de référence. Par rapport aux autres groupes, ils utilisent plus fréquemment leur téléphone pour appeler un membre de la famille (32\% de ce groupe l'utilisent à cette fin, contre $24 \%$ de l'ensemble des jeunes). $46 \%$ des jeunes du groupe des « casaniers paisibles ", contre $37 \%$ de l'ensemble des jeunes, disent rester plus fréquemment dans leur quartier.

Ici, l'entre-soi n'est pas seulement un repli, mais aussi une ressource pour affronter le monde extérieur. En effet, si le désir de ces lycéens du groupe des « casaniers paisibles » est de rester au plus près des proches, la norme qui consiste à tout mettre en œuvre pour trouver une place sur le marché de l'emploi apparaît, dans les entretiens, à travers l'investissement scolaire du groupe familial.

\subsection{La famille, un appui pour la réussite scolaire}

La plupart des lycéens qui se trouvent dans le groupe des " casaniers paisibles " et au sein de familles affiliées (Castel, 1991) relatent l'intérêt du groupe familial pour l'univers scolaire. Ils suivent un parcours plutôt continu et formulent assez précisément un projet professionnel.

L'investissement parental se mesure à l'aune du suivi et de l'aide aux devoirs. Cette aide, fortement structurante dans l'expérience positive de l'école (Lahire, 1995), est relatée comme un "ça va de soi " par ces jeunes:

"Et ton frère, il t'a aidé en cours? Ben oui, quand je ne comprends pas les cours d'histoire ou de maths, il m'explique, il est là. (...) Mais il maide si je demande. Mais en fait c'est plus avec mon père que je vois pour m'aider dans les cours de commerce et tout ça, car mon père, lui, il a fait un bac Comptabilité. Donc, tout ce qui est gestion et approvisionnement, il est là pour maider. " (Marie, 17 ans, est scolarisée en terminale bac pro Commerce à Tours. Elle habite avec ses parents et ses deux frères dans un pavillon situé dans la banlieue de Tours. Ses deux frères, âgés de 19 et 23 ans, sont diplômés d'un bac général, l'aîné travaille dans le commerce et le plus jeune étudie le droit à Tours. Ses parents ont toujours travaillé, son père est manager de stations-service et sa mère est chef vendeuse).

"Oui elle (sa mère) m'aidait un petit peu. Quand je ne comprenais pas, elle m'expliquait, je men souviens. (...) Au collège, elle a continué à maider. Et aujourd'hui, ça dépend quelle matière, sinon, on s'aide avec ma sour et mon père, ça dépend quelle matière. Si j'ai besoin, ils peuvent maider!" (Justine, 17 ans, est scolarisée en terminale bac pro Secrétariat à Châteauroux. Elle habite une maison avec ses parents et ses deux sœurs dans un village de 5000 habitants. Sa sœur aînée, 21 ans, est au chômage à la suite de la rupture d'un contrat d'apprentissage. Sa petite sœur de 13 ans est scolarisée. Ses parents, qui accèdent à la propriété, travaillent. Son père est agriculteur céréalier, sa mère assistante maternelle). 
Cette implication parentale dans la scolarité du jeune se retrouve dans les données issues de l'enquête par questionnaire. En effet, les jeunes du groupe des " casaniers paisibles " sont les lycéens les plus sollicités par leurs parents concernant leur parcours scolaire $(60 \%$ des jeunes sont sollicités par les deux parents au sujet de l'école, contre $46 \%$ de l'ensemble des jeunes).

À l'instar de leurs parents, ces jeunes ont également bien conscience que leur scolarité constitue une dimension importante de leur parcours de vie, conditionnant leur vie professionnelle et donc leur statut socio-économique futur. Ils savent également qu'elle sera d'autant plus valorisée et valorisable qu'elle sera dotée d'un diplôme supérieur au baccalauréat. Lorsque dans l'enquête quantitative, il est demandé aux jeunes du groupe des « casaniers paisibles » quelle activité ils exercent au domicile à leur retour du lycée, ils sont $35 \%$ à évoquer les devoirs scolaires, contre $28 \%$ sur l'ensemble de l'effectif. Contrairement à d'autres pairs, ces jeunes déterminés poursuivent donc leur voie et comptent bien se former davantage pour augmenter leurs chances d'insertion professionnelle future.

Les perspectives professionnelles de ces jeunes sont en phase avec leur formation actuelle. Ils établissent des liens entre leur formation actuelle et le métier qu'ils envisagent d'exercer. La stabilité professionnelle des parents favorise leur perception positive du travail. Ce dernier se présente comme le moteur de leur avenir, de la même manière qu'il est déjà moteur de ces foyers, tant il structure en grande partie l'organisation domestique et reste une référence identitaire pour ces groupes familiaux.

Etant ainsi disposés grâce aux positions héritées (parents ayant un emploi stable, ayant accédé à la propriété), et insérés dans un univers familial paisible et stable, qui permet d'être soutenu sur le plan scolaire, les jeunes sont en mesure de se projeter.

\subsection{L'éloignement pour accéder à l'emploi : le choix de la nécessité}

Les perspectives professionnelles ou de formation de ces lycéens peuvent les conduire à envisager un éloignement d'avec leur famille, amis et ville d'origine, même si cela leur coûte affectivement. Cette éventualité, accueillie avec plus ou moins d'appréhension, est d'autant plus acceptable qu'il y a un proche qui demeure là où ils souhaitent s'installer. Justine illustre cette situation :

"Je vais le tenter (Concours IRA $\left.{ }^{10}\right)$. J'ai regardé un peu sur Internet. C'est dans des grandes villes, je crois qu'il y a Orléans, si ce n'est pas Orléans, c'est Tours. Il y a aussi Paris. Il n'y en a pas à Châteauroux. Sinon, c'est dans le Sud, mais là non. (...) À Paris, c'est trop grand et Orléans ou Tours, je connais. J'ai une de mes tantes qui habite Orléans et j'en ai une autre qui habite à Tours."

10. Les IRA (Instituts Régionaux d'Administration) forment des attachés d'administration pour les différents services de l'État. 
La ville, espace de l'anonymat et de l'individualité (Simmel, 1989), inquiète. Être au loin en ayant un proche près de soi rassure. La réalité des territoires en termes d'offre de formation et d'opportunités d'emploi intervient également. Alors que Justine sait que son projet nécessite un éloignement, Marie n'envisage pas de partir dans la mesure où l'offre locale lui permet de conserver ses liens forts :

"Et tu envisages de faire quoi après ton Bac?"

"Un BTS (brevet de technicien supérieur) en alternance, ici, un BTS MUC (Management des Unités Commerciales). Qui est juste à côté. C'est deux ans, si je ne me trompe pas. Et les cours, c'est pas ce qui me passionne le plus, donc moi, je préfere être sur le terrain que de suivre des cours. Ben, j'ai des amis, mais ils veulent s'arrêter au Bac, et moi, je me dis que si je m'arrête au $B a c$, enfin, avec juste un Bac, on s'en sort pas. Et donc, du coup, moi, je préfere avancer dans mes études"

"(...) «On est tous chez nos parents. Ben, on est bien chez eux (rires). (...) J'aime Tours surtout pour les gens qui y sont, il y a tous mes potes, ma famille."

Plus que la peur de l'éloignement, ces jeunes nous disent la force de l'injonction au départ, la nécessaire mobilité pour "réussir ", et leur propension à s'y conformer. Bien entendu, le départ est plutôt douloureux, et s'il peut y avoir une accroche affective là où l'on s'installe, la décohabitation est plus facile. Ces jeunes ont besoin de trouver ce type d'appui pour sortir du " nid » protecteur. Ils ont aussi besoin de l'appui parental, notamment en termes économiques: "[mes parents] Ils mont dit si tu veux continuer, on te paiera tes études. Ah ben moi, je suis prête à partir. Si je dois partir, ben je m’en vais. " (Justine)

Bien qu'ils soient très proches de leur groupe familial et que le départ ne soit pas véritablement souhaité, leur conformité vis-à-vis des injonctions à l'emploi et à la mobilité semble rendre possible l'éloignement du foyer. Il en est tout autrement pour les jeunes qui vivent au sein d'un groupe familial fragilisé, dont les parents sont éloignés de la norme scolaire et de la norme emploi.

\section{Rester ou partir, un enjeu pour se défaire d'un groupe familial inhibant}

Ainsi, certains jeunes relevant du groupe des " casaniers paisibles " ne sont pas désireux de quitter leur domicile pour l'emploi, et expriment une distance vis-à-vis de la norme scolaire et de la norme emploi. D'autres jeunes, principalement au sein du groupe des " aventuriers sous tension ", désirent partir pour échapper à l'emprise du groupe familial. Ces deux cas de figure révèlent la prédominance d'une relation forte du jeune à ses parents, qui tend à le fixer au sein du groupe familial. Pour présenter ces deux cas de figure, nous nous appuierons sur deux récits, celui de Laurène et de sa mère, et celui de Nathalie. 


\subsection{Le repli dans l'entre-soi du groupe familial}

Lorsque le groupe familial est économiquement et socialement fragilisé, les relations en son sein tendent à se développer sur un mode fusionnel (Kellerhals, Montandon, 1991). Le monde extérieur, vécu comme hostile, porteur des normes et valeurs dominantes de la réussite scolaire et de la norme emploi, ne fait plus sens. Dans le cadre de sa socialisation, le jeune est alors plus enclin à s'éloigner du désir-maître des institutions. Cette distance à la norme scolaire et à la norme emploi et ses effets sur l'aspiration à la mobilité sont caractéristiques de l'histoire de Laurène.

Laurène est âgée de 15 ans lorsque nous la rencontrons en 2012. Elle habite avec ses parents et sa sœur aînée un petit village situé à $30 \mathrm{~km}$ de Tours, dans une petite ferme en location située dans les champs. Scolarisée en seconde professionnelle "Accueil » à Tours, Laurène abandonne sa formation un mois après son inscription, pour se réorienter en apprentissage "Vente ", mais elle renoncera tout autant à cette voie. Née d'une famille recomposée, elle est la plus jeune des trois enfants issus de cette recomposition. Son père, âgé de 66 ans, est à la retraite. Muni d'un CAP couvreur, il a toujours exercé ce métier depuis ses 14 ans. Cependant, un grave accident de travail, en 1999, l'a éloigné de l'emploi. Sa mère, âgée de 50 ans, n'a pas de diplôme. Elle a arrêté ses études en fin de $3^{\text {ème }}$ en raison de problèmes familiaux et a toujours réalisé des petits boulots comme employée de ménage. Des problèmes de santé réguliers invalident sa quête d'emploi.

Laurène est socialisée dans un cadre familial où les institutions sociales sont placées en extériorité par rapport à la famille. Dans le même temps, elle reçoit sa reconnaissance sociale principalement du groupe familial. Le premier extrait d'entretien met en évidence la façon dont la norme scolaire est appréhendée par le couple mère-fille :

Laurène : "Une fois, je ne comprenais pas un exercice, je lui ai demandé de m'expliquer (il s'agit du professeur de physique-chimie) encore mieux ce qu'elle avait expliqué à tout le monde, parce que je n'arrivais pas à comprendre. Je ne suis pas très forte en physique chimie et elle m'a dit, enfin, elle m'a fait comprendre que si je ne comprenais pas, c'est que je n'étais pas intelligente quoi! Ben du coup, ben voilà. "

Sa mère : "Oui, enfin, toi, tu ne mâches pas tes mots non plus. "

Laurène : "Ben non, mais enfin. "

Sa mère : "Ça, c'est clair qu'un prof qui lui manque de respect, elle ne se laisse pas faire! Elle a un caractère qui ne se laisse pas faire! Oui enfin, si la prof n'était pas respectueuse, ça amène de l'irrespect aussi!"

Laurène : "Ben oui, si ils disent..."

Sa mère : "Non, mais elle est jeune, donc c'est facile " 
Lorsque Laurène énonce que son professeur a invalidé ses compétences, elle est soutenue par sa mère qui ne remet pas en question les dires de sa fille. Il ne s'agit pas ici de faire le point avec l'enseignant, mais de justifier le comportement de sa fille face à "l'irrespect » du professeur. Il n'est pas question ici de renouer avec l'institution pour permettre la réussite de Laurène. Le monde familial et le monde scolaire sont imperméables l'un à l'autre. Il en est de même pour le monde du travail. Lors de son arrivée sur le lieu de travail, Laurène fait l'objet de remarques de la part de son employeur :

Laurène : "Oui, déjà le premier jour, je suis arrivée avec mon piercing, elle me dit: "Il va falloir menlever ça!" je lui dis: "Je suis désolée, c'est un cadeau d'anniversaire, ça coûte 50-60 euros, je ne vais pas l'enlever!"”

Sa mère : Tu l'as changé combien de fois ?"

Laurène : "Je l'ai changé trois ou quatre fois. "

(...) Enquêtrice: "Mais quand vous vous êtes entretenue avec elle la première fois, elle avait vu que vous aviez un piercing?"

Laurène : "Oui, ben oui, et elle m'a dit "Il faut le changer!"”

Sa mère : "On en a acheté trois pour arriver à la satisfaire, et même sur les trois, il y en a pas un qui l'a satisfait. Donc déjà ça et..."

Laurène: "Parce qu'elle voyait qu’une des vendeuses qui est déjà embauchée là-bas depuis longtemps, qui est en CDI et tout. Elle a un piercing au nez, mais c'est un piercing plat. Donc elle m'a dit: "Oui, vous devez mettre un piercing plat comme l'autre vendeuse". Je lui ai dit: "Je suis désolée, mais au Labret, il n’y a pas de piercing plat, ça n'existe pas, c'est que des trucs comme ça ou des pics, donc c'est pas mieux!"”

(...) Sa mère: "Oui, et puis après, à cause du chien (Laurène s'est fait mordre par son chien), tu as fait une inflammation en plus. "

Laurène : "Oui en plus. "

Sa mère : "Ben oui, comme elle a forcé dessus le jour même, elle a travaillé, travaillé, travaillé. Ben c'est sûr que l'inflammation est arrivée, infection par-dessus, donc elle a été arrêtée pendant 15 jours!"

Laurène et sa mère trouvent " normal » d'imposer une tenue à l'employeur. Ici, il n'est pas question d'avoir à se plier à la norme qui consiste à devoir se soumettre au désir de l'employeur pour s'assurer la conservation de l'emploi. S'il est possible de faire quelques efforts, cela ne doit pas être contraire à la volonté d'agir librement. Ce refus d'être sous la contrainte de l'autre est incorporé. L'arrêt de travail, suite à une infection, devient tout à fait légitime. Comme pour l'institution scolaire, le monde du travail est un monde à côté, dénué d'attractivité et marqué par la servitude. 
La distance des comportements aux normes scolaires et aux normes du monde du travail, telle que celle qui vient d'être présentée, tend à limiter la mise à distance du groupe familial pour trouver un emploi. Toutefois, le repli dans l'entre-soi familial peut devenir source de tensions.

\subsection{L'émancipation incertaine}

Certains jeunes expriment le souhait de quitter le groupe familial. De l'analyse des données issues du questionnaire à propos de l'usage du téléphone, il apparaît que $17 \%$ des jeunes du groupe des " aventuriers sous tension " ont tendance à utiliser leur téléphone pour appeler un membre de la famille contre, $24 \%$ de l'ensemble des jeunes. De même, lorsqu'il s'agit de rencontrer d'autres jeunes, $25 \%$ des jeunes du groupe des " aventuriers sous tension ", contre $37 \%$ de l'ensemble des jeunes, disent rester plus fréquemment dans leur quartier.

Lorsque les relations au sein de la famille sont décrites comme paisibles, les lycéens aspirant au départ du " nid " familial (groupe " des aventuriers paisibles ») sont moins enclins à limiter les contacts avec la famille et à établir des relations avec d'autres jeunes en dehors du quartier, sans que cela soit significatif par rapport à l'ensemble des jeunes.

Ici, la tension au sein de la famille constitue une modalité essentielle. En effet, la forte aspiration à trouver un emploi loin du domicile parental est plus l'expression d'une volonté de mettre une distance vis-à-vis du groupe familial que d'accéder à tout prix à l'emploi.

Lorsque les relations familiales sont plutôt de nature conflictuelle, mais que les relations affectives ont une forte emprise sur le jeune, celui-ci se sent obligé de rester au sein du foyer, même s'il aspire par ailleurs à partir. L'histoire de Nathalie illustre ce processus.

La mère de Nathalie ne travaille pas et souffre de problèmes psychiatriques. Elle a fait plusieurs tentatives de suicide. Son père, chauffeur-routier, souvent absent, décède prématurément lorsque Nathalie a 6 ans. Ses parents sont locataires et changent plusieurs fois de logement pour essayer d'obtenir de meilleures conditions de vie au fur et à mesure que la famille s'agrandit (Nathalie a un frère et une sœur plus jeunes qu'elle, qui seront placés en famille d'accueil après le décès du père). Nathalie établit d'ellemême une relation de cause à effet entre l'instabilité familiale (attribuée aux tentatives de suicide de sa mère) et ses difficultés scolaires. Elle a tenté un projet d'émancipation familiale en s'inscrivant dans une formation loin de sa famille et en vivant en internat, mais les difficultés de sa mère la ramènent à son point de départ. La famille et ses dysfonctionnements "polarisent " le projet de Nathalie et le restreignent à la ville de Châteauroux. À plus long terme, Nathalie sait, de par ses expériences précédentes, qu'il sera difficile de " lâcher " une famille dont le père est décédé, la mère suicidaire, le beau-père peu investi, les frères et sœurs placés, déficients mentaux. 
Nathalie: "Parce que j'ai vu que maintenant, ben je me suis aperçue que c'était, ça faisait loin Loches-Châteauroux et je me suis dit ben non, je préfere rester au plus près quand même de ma famille, même si je préférerais déjà partir encore plus loin... Voilà, parce que jai une vie familiale, c'est vraiment assez difficile tout ça. Donc, j’aimerais déjà moi partir, même si j’ai que 17 ans. Prendre mon envol et dire "ciao, je m'en vais"”.

Enquêteur : "Hum, mais à la fois tu..., tu voudrais partir... »

Nathalie : "Voilà..."

Enquêteur : "Mais tu reviens au plus près... "

Nathalie : "C'est ça.»

Enquêteur : "Et comment tu te l'expliques, ça ?À la fois tu veux partir et tu reviens?"

Nathalie: «Ben, ça reste ma famille quand même. Donc, on peut pas couper les ponts avec sa famille, sachant quils ont toujours été là quand javais besoin."

Enquêteur : "Ouais .»

Nathalie : "Mais d'un côté, plus vite je pourrai partir, plus vite je m’en irai."

(Nathalie, 22ans, scolarité arrêtée en $2^{\text {nde }}$ année de bac pro Aide à la personne, parents séparés).

Nathalie évoque son projet de mobilité contrarié par les problèmes de santé physique et psychique de sa mère et par la faiblesse du soutien de la famille. Son projet peut être lu comme un système de tension entre le " je " et le "nous ", entre un désir de réalisation individuelle et un sens du devoir fortement ancré. L'usage du pronom impersonnel "On " ("On ne peut pas couper les ponts") informe sur la norme intériorisée par Nathalie, alors que tous les désirs de partir sont exprimés à la première personne du singulier: "Plus vite je pourrai partir, plus vite je m’en irai ». La proximité vis-à-vis du domicile familial est associée à la contrainte, alors que l'éloignement est porteur de liberté. Au moment de l'entretien, Nathalie est confrontée à cette contradiction et ne parvient pas à la résoudre.

\section{Conclusion}

L'étude des flux migratoires des diplômés au moment de l'insertion professionnelle ne saurait se limiter à l'analyse de l'adéquation des formations au marché du travail local. En interrogeant des lycéens en formation professionnelle, plutôt que des diplômés, et en diversifiant leurs caractéristiques sociales et spatiales, la recherche a permis de mettre en évidence l'importance de la socialisation familiale dans la manière d'envisager l'éloignement du groupe familial pour occuper un premier emploi. La possibilité d'un éloignement dépend conjointement de trois facteurs : la stabilité de l'intégration professionnelle des parents, leur degré de conformité avec le désir-maitre des institu- 
tions sociales (dont l'école), et l'existence ou non de relations soutenues et affectueuses $\mathrm{du}$ jeune avec ses proches (famille, amis).

Si les jeunes titulaires d'un baccalauréat professionnel tendent progressivement à différer leur entrée sur le marché de l'emploi en poursuivant des études supérieures, conformément aux objectifs fixés par les pouvoirs publics, il n'en demeure pas moins que l'éloignement du groupe familial pour suivre ces études mériterait d'être étudié en tenant compte des trois facteurs précités.

\section{Bibliographie}

Baron M., Perret C. (2006), "Bacheliers, étudiants et jeunes diplômés : quels systèmes migratoires régionaux ? ", L'Espace géographique, 1, pp. 44-62.

Beaud S., Pialoux M. (1999), Retour sur la condition ouvrière, Paris, Fayard.

Blanchet A., Gotman A. (1992), L'enquête et ses méthodes : l'entretien, Paris, Armand Colin, coll. « $128 »$.

Caro P. (2006), "La dimension spatiale des systèmes formation-emploi ", L'espace géographique, $\mathrm{n}^{\circ} 3$, Tome 35, pp. 223-240.

Castel R. (1989), « La question sociale commence en 1349 ", Vie sociale, mai, pp. 9-25.

Castel R. (1991), " De l'indigence à l'exclusion, la désaffiliation », in Donzelot Jacques (sous la dir.), Face à l'exclusion, le modèle français, Paris, Éditions Esprit, pp. 137-168.

Castel R. (1995), Les métamorphoses de la question sociale. Une chronique du salariat, Paris, Fayard.

Couet C. (2006), "La mobilité résidentielle des jeunes ", Données sociales - La société française, pp. 495-504.

Demazière D., Dubar C. (1997), Analyser les entretiens biographiques - l'exemple des récits d'insertion, Nathan.

Didier-Fèvre (2014), "Les jeunes de l'espace périurbain à l'épreuve des choix post-bac ", Formation Emploi, $\mathrm{n}^{\circ} 127$, juillet-septembre, pp. 27-48.

Durville J.-M., Connin P. (2009), "La région Centre, terre de migration», Insee Centre Info, $\mathrm{n}^{\circ} 156$, septembre.

Esterle-Hedibel M. (2006), "Absentéisme, déscolarisation, décrochage scolaire, les apports des recherches récentes ", Déviance et Société, 2006/1, vol. 30, pp. 41-65.

Freyssinet J. (2004), Le chômage, Paris, La Découverte, coll. « Repères ». 
Geay B. (2003), « Du 'cancre' au 'sauvageon' - Les conditions institutionnelles de diffusion des politiques 'd'insertion' et de 'tolérance zéro' ", Actes de la Recherche en Sciences Sociales, $\mathrm{n}^{\circ} 149$, pp. 21-31.

Geremek. B. (1987), La potence ou la pitié - L'Europe et les pauvres du moyen-âge à nos jours, Paris, Gallimard.

Jellab A. (2008), Sociologie du lycée professionnel. L'expérience des élèves et des enseignants dans une institution en mutation, Toulouse, Presses universitaires du Mirail.

Kellerhals J., Montandon C. (1991), Les stratégies éducatives des familles, Neuchâtel, Delachaux et Niestlé.

Lahire B. (1995), Tableaux de familles, Paris, Hautes études, Gallimard, Seuil.

Lordon F. (2010), Capitalisme, désir et servitude, Paris, La Fabrique.

Mauger G. (2013), « Bourdieu et les classes populaires. L'ambivalence des cultures dominées ", in Coulangeon P., Duval J., Trente ans après la distinction de Pierre Bourdieu, Paris, La Découverte, coll. "Recherches », pp. 243-254.

Péan S. (2012), "Les bacheliers professionnels dans l'enseignement supérieur ", Note d'information, Enseignement supérieur et Recherche, 12.04, pp. 1-8.

Perret C. (2007), «Quitter sa région pour entrer à l'université : quels sont les facteurs explicatifs de la mobilité géographique des bacheliers ? ", Orientation Scolaire et professionnelle, 36, $\mathrm{n}^{\circ}$ 3, pp. 313-342.

Retière J.-N. (2003), "Autour de l'autochtonie. Réflexions sur la notion de capital social populaire ", Politix, Vol. 16, n 63, pp. 121-143.

Simmel G. (1989), "Les grandes villes et la vie de l'esprit ", in Simmel G., Philosophie de la modernité, Paris, Payot, pp. 233-252 (1903).

Schwartz O. (1990), Le monde privé des ouvriers - Hommes et femmes du nord, Paris, PUF. 